\title{
Programa computacional para dimensionamento e avaliação de sistemas de irrigação por sulco
}

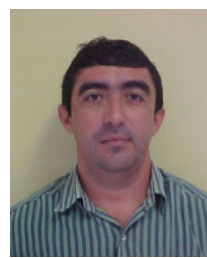

\author{
Aderson S. de Andrade Júnior ${ }^{1}$, Valdemício F. de Sousa ${ }^{2}$, Cristina M. Alencar ${ }^{3}$, Silvana da S. Cardoso ${ }^{4} \&$ José A. Frizzone $^{5}$ \\ 1 Embrapa Meio Norte, CP 01, CEP 64.006-220, Teresina, PI. E-mail: aderson@cpamn.embrapa.br (Foto) \\ 2 Embrapa Meio Norte, Teresina, PI \\ ${ }^{3}$ DER/UNESP, CEP 18603-970,Botucatu, SP. Fone: (14) 6802-7165 \\ ${ }^{4}$ DER/ESALQ/USP, CEP 13418-900, Piracicaba, SP. Fone: (19) 3429-4217 \\ 5 DER/ESALQ/USP, CEP 13418-900, Piracicaba, SP. Fone: (19) 3429-4217. E-mail: frizzone@carpa.ciagri.usp.br
}

Protocolo $019-22 / 02 / 2000$

\begin{abstract}
Resumo: Este trabalho apresenta o aplicativo denominado DimSulco, desenvolvido em linguagem Visual Basic 5, em ambiente Windows 95, que objetiva facilitar ao usuário o dimensionamento e a avaliação de sistemas de irrigação por sulco, a partir do conhecimento prévio de parâmetros básicos de projeto. O DimSulco foi estruturado em cinco módulos: I - Irrigação sem déficit hídrico e comprimento do sulco definido; II - Irrigação sem déficit hídrico e comprimento do sulco indefinido; III - Irrigação com déficit hídrico e comprimento do sulco definido; IV Irrigação com déficit hídrico e comprimento do sulco indefinido e $V$ - Sistema de reutilização de água. Os módulos I e III foram divididos em dois submódulos, que permitem o dimensionamento e a avaliação do sistema com e sem vazão reduzida. Os módulos II e IV foram divididos em quatro submódulos, contemplando as seguintes opções: a) sem vazão reduzida e tempo de avanço (Ta) igual a 1/4 do tempo de oportunidade de infiltração da lâmina requerida no final da parcela (To); b) com vazão reduzida e Ta igual a $1 / 4$ de To; c) sem vazão reduzida e Ta igual a To e d) com vazão reduzida e Ta igual a To. $O$ módulo $V$ dimensiona sistemas de reutilização da água a partir de um dimensionamento prévio efetuado em qualquer um dos módulos anteriores, definindo os elementos básicos de cada setor e recalculando os índices de desempenho. Para ilustrar a execução do programa, utilizou-se um exemplo de dimensionamento de um sistema de irrigação por sulco.
\end{abstract}

Palavras-chave: aplicativo agrícola, sistema de reutilização da água, irrigação com déficit

\section{Software for designing and evaluation of furrow irrigation systems}

\begin{abstract}
This work presents a software named DimSulco, developed in the Visual Basic language, version 5 for Windows 95, with the objective of helping the user to design and to evaluate furrow irrigation systems from previous knowledge of fundamental project parameters. It was structured in five modules: I - Without deficit irrigation and defined furrow length; II - Without deficit irrigation and undefined furrow length; III - Deficit irrigation and defined furrow length; IV - Deficit irrigation and undefined furrow length and V - Reuse of irrigation runoff system. The modules I and III are divided in two sub-modules, which allow the design and performance of the system with and without cutback inflow. The modules II and IV are divided in four sub-modules including the options: a) Without cutback inflow and advance time (Ta) equal to $1 / 4$ opportunity time for infiltration of the depth of water at the final end (To); b) With cutback inflow and Ta equal to $1 / 4$ To; c) Without cutback inflow and Ta equal to To and d) With cutback inflow and $\mathrm{Ta}$ equal to To. The module $\mathrm{V}$ allows the design of irrigation runoff reuse systems using any of the previously designed modules, calculating the basic elements of the each set and performance of irrigation variables. To illustrate an application of this software one example of a furrow irrigation system was used.
\end{abstract}

Key words: agricultural software, reuse of irrigation runoff system, deficit irrigation 


\section{INTRODUÇÃO}

Os sistemas de irrigação por superfície predominam na maioria das áreas irrigadas do mundo, porém, o uso inadequado e o desconhecimento de suas potencialidades têm resultado em reduzida eficiência de aplicação de água; no entanto, quando adequadamente dimensionados e manejados, podem apresentar desempenho satisfatório e comparável a qualquer outro sistema de irrigação (Scaloppi, 1986; Souza \& Scaloppi, 1998).

O dimensionamento desses sistemas pode ser efetuado através de um processo de tentativas realizado no campo ou se utilizando programas computacionais. Neste particular, os aplicativos constituem excelente ferramenta, uma vez que possibilitam inúmeras alternativas de dimensionamento a um custo e tempo reduzidos (Frizzone, 1993). No entanto, existem poucos aplicativos nacionais (Rivera et al., 1997; Vilas Boas \& Scaloppi, 1998) e planilhas eletrônicas (Azevedo et al., 1998) que efetuem o dimensionamento e a avaliação de sistemas de irrigação por superfície, os quais apresentam diferenças quanto à linguagem de programação utilizada, ao grau de complexidade, precisão e parâmetros requeridos para processamento. Além disso, os referidos aplicativos não contemplam o dimensionamento nem a avaliação sob condição de irrigação com déficit hídrico.

Neste trabalho apresenta-se o aplicativo DimSulco, que constitui uma ferramenta auxiliar simples e de fácil interação com o usuário, visando efetuar o dimensionamento e a avaliação de sistemas de irrigação por sulco com drenagem livre, a partir do conhecimento prévio de parâmetros básicos de projeto.

\section{DESENVOLVIMENTO}

O programa computacional DimSulco* foi desenvolvido em linguagem de programação Visual Basic 5, em ambiente Windows 95, estruturado em cinco módulos: I - Irrigação sem déficit hídrico e comprimento do sulco definido; II Irrigação sem déficit hídrico e comprimento do sulco indefinido; III - Irrigação com déficit hídrico e comprimento do sulco definido; IV - Irrigação com déficit hídrico e comprimento do sulco indefinido e V - Sistema de reutilização de água.

Os módulos I e III foram divididos em dois submódulos, que permitem o dimensionamento e a avaliação do sistema com e sem vazão reduzida. Assim, somente é possível uma redução de vazão, calculada através do método do somatório das infiltrações parciais (Bernardo, 1989) ou vazões parciais (Frizzone, 1993). Os módulos II e IV foram divididos em quatro submódulos, considerando-se as opções com e sem redução de vazão e a relação entre os tempos de avanço $(\mathrm{Ta})$ e de oportunidade de infiltração da lâmina requerida no final da parcela (To): a) sem vazão reduzida e Ta igual a 1/1/4 de To; b) com vazão reduzida e Ta

* Cópia gratuita do programa pode ser solicitada ao primeiro autor igual a 1/4 de To; c) sem vazão reduzida e Ta igual a To e d) com vazão reduzida e Ta igual a To. Nas opções a e b, o cálculo do comprimento do sulco é realizado pelo critério tradicional de Criddle, segundo o qual a frente de avanço deveria atingir o final da parcela em $1 / 4$ do tempo total de aplicação da lâmina de irrigação requerida (Scaloppi, 1986). Nas opções c e d utilizou-se o critério em que o comprimento do sulco é definido pela equivalência entre os tempos de avanço e de oportunidade de infiltração (Frizzone, 1993). O módulo $\mathrm{V}$ efetua o dimensionamento de um sistema de reutilização da água a partir do dimensionamento prévio efetuado em qualquer um dos módulos anteriores, definindo os elementos básicos de cada setor e recalculando os índices de desempenho. Os dados de entrada solicitados são: a) parâmetros da função de avanço ( $\mathrm{K} \mathrm{e} \mathrm{b})$ e perímetro molhado; b) parâmetros da função de infiltração ( $\beta, \alpha$ e fo) e perímetro molhado e c) parâmetros do projeto: módulo I comprimento do sulco (m), espaçamento entre sulcos (m), vazão disponível $\left(\mathrm{L} \mathrm{s}^{-1}\right)$, vazão derivada ao sulco $\left(\mathrm{L} \mathrm{s}^{-1}\right)$, tempo de recesso ( $\mathrm{min})$, lâmina de irrigação requerida $(\mathrm{mm})$, evapotranspiração da cultura $\left(\mathrm{mm} \mathrm{d}^{-1}\right)$, largura da área $(\mathrm{m})$ e comprimento da área (m); módulo II - igual ao módulo I, excluindo-se o comprimento do sulco, o qual será um parâmetro de saída do dimensionamento; módulo III - igual ao módulo I, adicionando-se o nível de déficit hídrico desejado (\%); módulo IV - idêntico ao módulo II, acrescentando-se o nível de déficit hídrico desejado (\%) e módulo $\mathrm{V}$ - parâmetros da função de infiltração ( $\beta, \alpha$ e fo), vazão derivada ao sulco $\left(\mathrm{L} \mathrm{s}^{-1}\right)$, vazão disponível $\left(\mathrm{L} \mathrm{s}^{-1}\right)$, tempo de corte (min), perda de água por escoamento superficial do dimensionamento sem sistema de reutilização de água (SRE) (\%), número de setores sem SRE, comprimento da área $(\mathrm{m})$, largura da área $(\mathrm{m})$, lâmina de irrigação requerida $(\mathrm{mm})$ e espaçamento entre sulcos $(\mathrm{m})$.

$O$ resultado do dimensionamento e os índices de desempenho calculados, são: a) Dimensionamento - tempo de corte (min), número total de setores, número de sulcos irrigados por setor, número de setores irrigados por dia, comprimento do setor (m), largura do setor (m), área irrigada por setor (ha), área irrigada total (ha), turno de irrigação (d), período de irrigação (d), período efetivo de irrigação (d) e jornada de trabalho diário (h) e b) Índices de desempenho - uniformidade de distribuição (\%), eficiência de aplicação (\%), eficiência de armazenamento (\%), perda por percolação (\%) e perda por escoamento superficial (\%). Nas opções que envolvem redução de vazão, são adicionalmente apresentados: vazão reduzida ótima $\left(\mathrm{L} \mathrm{s}^{-1}\right)$, tempo de irrigação com vazão reduzida (min) e tempo de irrigação com vazão derivada (min). Nos submódulos com comprimento do sulco indefinido é acrescentado o comprimento do sulco (m).

No módulo V são calculados: número de setores, número de sulcos por setor, área de cada setor (ha), volume escoado e retornado a cada setor $\left(\mathrm{m}^{3}\right)$, vazão retornada a cada setor $\left(\mathrm{L} \mathrm{s}^{-1}\right)$, volume do reservatório $\left(\mathrm{m}^{3}\right)$, volume residual $\left(\mathrm{m}^{3}\right)$, volume recuperado (\%), déficit hídrico no último setor (\%), eficiência de aplicação (\%), perda por escoamento superficial 
(\%) e perda por percolação profunda (\%). As equações matemáticas utilizadas no dimensionamento e avaliação do sistema são apresentadas em Frizzone (1993).

\section{APLICAÇÃo}

Para ilustrar a execução do programa utilizou-se um exemplo de dimensionamento de um sistema de irrigação por sulco, com as características abaixo relacionadas, assumindo-se uma situação de irrigação sem déficit hídrico, com comprimento do sulco definido e com as opções de uso ou não de vazão reduzida: parâmetros da função de avanço $(\mathrm{K}=0,0046 ; \mathrm{b}=2,023 \mathrm{e}$ $\mathrm{Pm}=0,21 \mathrm{~m})$; parâmetros da função de infiltração $(\beta=0,00124$; $\alpha=0,535$; fo $=0,00019 \mathrm{~m} \mathrm{~min}^{-1} \mathrm{e} \mathrm{Pm}=0,26 \mathrm{~m}$ ); comprimento do sulco $=100 \mathrm{~m}$ e $200 \mathrm{~m}$; espaçamento entre sulcos $=0,9 \mathrm{~m}$; vazão disponível $=110 \mathrm{~L} \mathrm{~s}^{-1}$; vazão derivada ao sulco $=0,8 \mathrm{~L} \mathrm{~s}^{-1}$; tempo de recesso $=10 \mathrm{~min}$; lâmina de irrigação requerida $=50 \mathrm{~mm}$; evapotranspiração da cultura $=5 \mathrm{~mm} \mathrm{~d}^{-1}$; largura da área $=400 \mathrm{me}$ comprimento da área $=500 \mathrm{~m}$, cujos resultados são apresentados na Tabela 1.

Verificou-se que, para o comprimento de sulco de $100 \mathrm{~m}$ e vazão reduzida de $0,417 \mathrm{~L} \mathrm{~s}^{-1}$, os índices de desempenho relativos à eficiência de aplicação (Ea) e perda por escoamento superficial $(\mathrm{Pe})$ foram melhores quando comparados à não redução de vazão, uma vez que esta condição promove redução significativa das perdas por escoamento superficial no final da parcela. Com relação ao comprimento de sulco de $200 \mathrm{~m}$, a utilização da vazão reduzida de $0,689 \mathrm{~L} \mathrm{~s}^{-1}$ durante o intervalo de tempo de 146,3 min, não foi tão vantajosa em comparação à situação anterior, devido ao pequeno acréscimo na eficiência de aplicação (de 48,8 para 51,5\%) em função de uma ligeira diminuição nas perdas por escoamento superficial (de 23,0 para $18,7 \%$ ).

Neste exemplo, deve-se optar pelo comprimento de sulco de $100 \mathrm{~m}$, devido ao seu melhor desempenho. Havendo interesse em melhorar ainda mais os índices de desempenho do sistema, a alternativa aconselhada seria a utilização da irrigação com déficit hídrico, associada à prática da redução de vazão ou o dimensionamento de um sistema de reutilização de água (Tabela 2).

Constatou-se que a adoção do sistema de reutilização de água melhorou bastante os índices de desempenho, notadamente quando se optou por sulcos com comprimento de 100 m (Tabela 2). Para esta opção de comprimento de sulco, a eficiência de aplicação de água foi praticamente a mesma, com e sem utilização de vazão reduzida; contudo, o uso da vazão reduzida proporcionou menor perda por escoamento superficial e requer um reservatório de menor capacidade, acarretando diminuição de custos com obras e equipamentos necessários ao bombeamento da água de escoamento.

A opção de sistema de reutilização para sulcos com comprimento de $200 \mathrm{~m}$, com e sem uso de vazão reduzida, não se mostrou viável tecnicamente, uma vez que os índices de desempenho não apresentaram melhoria significativa, além de comprometer a produção da cultura a ser irrigada pela imposição de déficits hídricos elevados (30 e 57\%) no último setor do sistema (Tabela 2).

Logicamente, outras análises semelhantes poderão ser realizadas com as outras possibilidades de dimensionamento disponíveis no programa, obtendo-se resultados imediatos pela alteração nos valores dos dados de entrada.

Tabela 1. Dimensionamento e índices de desempenho de um sistema de irrigação por sulco, assumindo-se situação de irrigação sem déficit, com comprimento do sulco (Cs) definido e com ou sem redução de vazão

\begin{tabular}{|c|c|c|c|c|}
\hline \multirow{2}{*}{ Parâmetros } & \multicolumn{2}{|c|}{ Sem redução de vazão } & \multicolumn{2}{|c|}{ Com redução de vazão } \\
\hline & $\mathrm{Cs}=100 \mathrm{~m}$ & $\mathrm{Cs}=200 \mathrm{~m}$ & $\mathrm{Cs}=100 \mathrm{~m}$ & $\mathrm{Cs}=200 \mathrm{~m}$ \\
\hline \multicolumn{5}{|l|}{ Índices de desempenho (\%) } \\
\hline Uniformidade de distribuição & 96,6 & 78,2 & 96,6 & 78,2 \\
\hline Eficiência de aplicação & 41,2 & 48,8 & 63,2 & 51,5 \\
\hline Eficiência de armazenamento & 100,0 & 100,0 & 100,0 & 100,0 \\
\hline Perda por percolação & 5,3 & 28,2 & 8,2 & 29,8 \\
\hline Perda por escoamento superficial & 53,5 & 23,0 & 28,6 & 18,7 \\
\hline \multicolumn{5}{|l|}{ Dimensionamento } \\
\hline Tempo de corte $(\min )$ & 227,4 & 384,1 & 227,4 & 384,1 \\
\hline Vazão reduzida ótima $\left(\mathrm{L} \mathrm{s}^{-1}\right)$ & - & - & 0,417 & 0,689 \\
\hline Tempo com vazão reduzida (min) & - & - & 165,3 & 146,3 \\
\hline Tempo com vazão derivada (min) & - & - & 62,1 & 237,8 \\
\hline $\mathrm{N}^{o}$ total de setores & 16,0 & 8,0 & 16,0 & 8,0 \\
\hline $\mathrm{N}^{o}$ de sulcos irrigados setor ${ }^{-1}$ & 137,0 & 137,0 & 137,0 & 137,0 \\
\hline $\mathrm{N}^{o}$ setores irrigados $\mathrm{d}^{-1}$ & 2,0 & 1,0 & 2,0 & 1,0 \\
\hline Comprimento do setor (m) & 123,0 & 123,0 & 123,0 & 123,0 \\
\hline Largura do setor $(\mathrm{m})$ & 100,0 & 100,0 & 100,0 & 100,0 \\
\hline Área irrigada/setor (ha) & 1,2 & 1,2 & 1,2 & 1,2 \\
\hline Área total irrigada (ha) & 19,7 & 19,7 & 19,7 & 19,7 \\
\hline Turno de irrigação (d) & 10,0 & 10,0 & 10,0 & 10,0 \\
\hline Período de irrigação (d) & 8,0 & 8,0 & 8,0 & 8,0 \\
\hline Período efetivo de irrigação (d) & 8,0 & 8,0 & 8,0 & 8,0 \\
\hline Jornada de trabalho diária $(\mathrm{h})$ & 7,6 & 6,4 & 7,6 & 6,4 \\
\hline
\end{tabular}


Tabela 2. Dimensionamento e índices de desempenho de um sistema de irrigação por sulco, considerando-se um sistema de reutilização de água (SRE) com ou sem redução de vazão

\begin{tabular}{|c|c|c|c|c|}
\hline \multirow{2}{*}{ Parâmetros } & \multicolumn{2}{|c|}{ Sem redução de vazão } & \multicolumn{2}{|c|}{ Com redução de vazão } \\
\hline & $\mathrm{Cs}=100 \mathrm{~m}$ & $\mathrm{Cs}=200 \mathrm{~m}$ & $\mathrm{Cs}=100 \mathrm{~m}$ & $\mathrm{Cs}=200 \mathrm{~m}$ \\
\hline \multicolumn{5}{|l|}{ Índices de desempenho (\%) } \\
\hline Eficiência de aplicação & 89,8 & 64,2 & 89,7 & 64,2 \\
\hline Eficiência de armazenamento & 100,0 & 100,0 & 100,0 & 100,0 \\
\hline Perda por percolação & 5,3 & 28,2 & 8,2 & 29,8 \\
\hline $\mathrm{N}^{\circ}$ de SRE em operação & 4 & 2 & 4 & 2 \\
\hline $\mathrm{N}^{\mathrm{o}}$ de setores / SRE & 3 & 4 & 4 & 4 \\
\hline $\mathrm{N}^{\circ}$ total de setores & 12 & 8 & 16 & 8 \\
\hline Área total irrigada (ha) & 20 & 20 & 20 & 20 \\
\hline Volume do reservatório $\left(\mathrm{m}^{3}\right)$ & 1228 & 745 & 381 & 547 \\
\hline Volume residual $\left(\mathrm{m}^{3}\right)$ & 172 & 0 & 102 & 0 \\
\hline 137 & 1,2 & 800,03 & - & - \\
\hline 210 & 1,9 & 1228,05 & 796,81 & 58,4 \\
\hline 208 & 1,9 & 172,33 & 2270,36 & 166,4 \\
\hline \multicolumn{5}{|c|}{ Sistema de reutilização: $\mathrm{Cs}=100 \mathrm{~m}$ e com redução de vazão } \\
\hline 137 & 1,2 & 278,84 & - & - \\
\hline 176 & 1,6 & 358,59 & 277,55 & 20,3 \\
\hline 187 & 1,7 & 381,40 & 355,83 & 26,1 \\
\hline 55 & 0,5 & 101,93 & 391,41 & 28,7 \\
\hline \multicolumn{5}{|c|}{ Sistema de reutilização: $C s=200 \mathrm{~m}$ e sem redução de vazão } \\
\hline 137 & 2,5 & 580,94 & - & - \\
\hline 89 & 1,6 & 716,86 & 1554,16 & 67,4 \\
\hline
\end{tabular}

\section{CONCLUSÃO}

O programa DimSulco constitui-se em uma ferramenta auxiliar para o dimensionamento e avaliação de sistemas de irrigação por sulco, possibilitando ao usuário, a partir do conhecimento prévio dos parâmetros básicos de projeto, várias alternativas de dimensionamento em uma interface bastante simples e fácil.

\section{LITERATURA CITADA}

Azevedo, H.M.; Azevedo, C.A.V.; Dantas Neto, J. Planilha eletrônica para determinação de parâmetros de sulcos de irrigação abertos no final. In: Congresso Brasileiro de Engenharia Agrícola, 27, 1998, Poços de Caldas. Anais ... Lavras: UFLA/SBEA, 1998. v.2, p.299-301.

Bernardo, S. Manual de irrigação. Viçosa: UFV, Imprensa Universitária, 1989. cap. 7, p.297-365.
Frizzone, J.A. Irrigação por superfície. Piracicaba: ESALQ/USP, 1993. 183p. LER. Série Didática, 5

Rivera, R.N.C.; Souza, F.; Teixeira, A.S. "Software" para o dimensionamento de sistemas de irrigação por sulcos via balanço de volume. I: Desenvolvimento. Engenharia Rural, Piracicaba, v.8, n.2, p.1-72, 1997.

Scaloppi, E.J. Sistemas de irrigação por superfície. Informe Agropecuário, Belo Horizonte, v.12, n.139, p.12-26, 1986.

Souza, C.F.; Scaloppi, E.J. Desempenho do regime de vazão continuamente reduzido em irrigação por sulcos. In: Congresso Brasileiro de Engenharia Agrícola, 27, 1998, Poços de Caldas. Anais ... Lavras: UFLA/SBEA, 1998. v.1, p.244-246.

Vilas Boas, M.A.; Scaloppi, E.J. Programa computacional para simulação da irrigação por superfície. In: Congresso Brasileiro de Engenharia Agrícola, 27, 1998, Poços de Caldas. Anais ... Lavras: UFLA/SBEA, 1998. v.2, p.217-219. 